\title{
Augmentation with an ovine forestomach matrix scaffold improves histological outcomes of rotator cuff repair in a rat model
}

\author{
Matthew Street ${ }^{1}$, Ashvin Thambyah² ${ }^{2}$ Michael Dray ${ }^{3}$, Satya Amirapu ${ }^{4}$, Donna Tuari ${ }^{1}$, Karen E Callon ${ }^{1}$, \\ Julie D Mclntosh ${ }^{5,6}$, Kristina Burkert ${ }^{5,6}$, P Rod Dunbar ${ }^{5,6}$, Brendan Coleman ${ }^{7}$, Jillian Cornish ${ }^{1}$ and David S Musson ${ }^{1^{*}}$
}

\begin{abstract}
Background: Rotator cuff tears can cause significant pain and functional impairment. Without surgical repair, the rotator cuff has little healing potential, and following surgical repair, they are highly prone to re-rupture. Augmenting such repairs with a biomaterial scaffold has been suggested as a potential solution. Extracellular matrix (ECM)-based scaffolds are the most commonly used rotator cuff augments, although to date, reports on their success are variable. Here, we utilize pre-clinical in vitro and in vivo assays to assess the efficacy of a novel biomaterial scaffold, ovine forestomach extracellular matrix (OFM), in augmenting rotator cuff repair.
\end{abstract}

Methods: OFM was assessed in vitro for primary tenocyte growth and adherence, and for immunogenicity using an assay of primary human dendritic cell activation. In vivo, using a murine model, supraspinatus tendon repairs were carried out in 34 animals. Augmentation with OFM was compared to sham surgery and unaugmented control. At 6- and 12-week time points, the repairs were analysed biomechanically for strength of repair and histologically for quality of healing.

Results: OFM supported tenocyte growth in vitro and did not cause an immunogenic response. Augmentation with OFM improved the quality of healing of the repaired tendon, with no evidence of excessive inflammatory response. However, there was no biomechanical advantage of augmentation.

Conclusions: The ideal rotator cuff tendon augment has not yet been identified or clinically implemented. ECM scaffolds offer a promising solution to a difficult clinical problem. Here, we have shown improved histological healing with OFM augmentation. Identifying materials that offset the poorer mechanical properties of the rotator cuff post-injury/repair and enhance organised tendon healing will be paramount to incorporating augmentation into surgical treatment of the rotator cuff.

Keywords: Rotator Cuff, Arthroplasty, Augmentation, Biomaterial, Tissue engineering

\section{Introduction}

Rotator cuff tears are a common cause of significant shoulder pain and functional impairment and are the most common cause of shoulder-related disability [1]. They are estimated to affect $22 \%$ of the general population, and the prevalence significantly increases with age [2]. Furthermore, with our increasing and ageing population, the

\footnotetext{
* Correspondence: d.musson@auckland.ac.nz

'Department of Medicine, The University of Auckland, Private Bag 92019, Auckland 1142, New Zealand

Full list of author information is available at the end of the article
}

incidence of rotator cuff tears requiring surgical intervention is steadily increasing [3].

Due to the avascular and acellular nature of the rotator cuff, healing potential is limited without surgical repair [4-6]. In a rat model of rotator cuff tears, histological analysis showed that $78 \%$ of tendons had incomplete closure of the defect site at 12 weeks post defect creation, and those defects that were fully closed had disorganised tissue inferior to that of the normal tendon [4]. Even after surgical repair, healing is suboptimal. In human patients over the age of 65 , only $43 \%$ of patients showed evidence of healing in a computed tomographic arthrogram or 
magnetic resonance imaging scans 18 months after arthroscopic repair of full thickness rotator cuff tears, while $86 \%$ of patients under 65 years of age showed evidence of healing [7]. Furthermore, re-tear rates are reported to be as high as $41 \%$ for double row arthroscopic repairs and $69 \%$ for single row repairs of large full thickness rotator cuff tears $[8,9]$.

Recently, biological augmentation with tissue-engineered grafts has been suggested as a method for improving healing outcomes in grades 3 and 4 tears (repairable, full thickness, small to large tears) and as an interposition graft for grade 5 tears (large retracted tears that are unable to be apposed) [10]. These grafts can be used either as an onlay (reinforcement) augmentation, where the graft is placed over the repaired tendon, or as an intercalary (interpositional) augmentation, where the graft is placed between the bone and retracted tendon.

Augmentation has two potential benefits: (1) providing mechanical support and offloading the tendon at the time of initial repair and (2) improving the rate and/or quality of healing [11]. Therefore, the ideal graft would achieve both of these benefits with the added parameters that it has good suture retention properties and is immunologically inert and fully degradable in a biological setting.

Extracellular matrix (ECM)-based scaffolds are currently the most commonly used rotator cuff augments [11]. Most components of ECM are homogenous among different species allowing properly processed ECM xenografts to be used with minimal anti-host inflammation [12, 13]. Furthermore, ECM has a complex three-dimensional microstructure, involving structural proteins such as collagen, fibronectin and laminin, glycosaminoglycans (GAGs) and bioactive growth factors. Combined, these allow for a degree of mechanical support, but more importantly, provide host cell attachment sites and a reservoir of growth factors that encourage cell migration, proliferation and differentiation while also modulating angiogenesis and immune response [12].

Recently, clinical confidence in the use of ECM scaffolds received a significant setback when a porcine non-crosslinked SIS (Restore ${ }^{\mathrm{Tm}}$ ) was associated with hypersensitivity reactions in $20-30 \%$ of patients $[14,15]$. It has been suggested that this reaction was secondary to a large amount of foreign DNA retained in Restore $^{\mathrm{TM}}$ and led to the product being no longer recommended for use by the American Academy of Orthopaedic Surgeons $[15,16]$. Therefore, all potential scaffolds need to be thoroughly tested prior to clinical use and closely monitored following commercial release.

Decellularized ovine forestomach matrix (OFM) is an ECM scaffold produced from the lamina propria of the ovine forestomach. It is decellularized using proprietary processes that retain the native collagen structure and secondary molecules that associate with ECM [17]. OFM is used clinically as a dermal template in the treatment of chronic wounds [18-20], can act as a template for tissue engineered skin grafts [21] and has also been used successfully as split thickness skin grafts [22].

Through the studies presented here, we aim to assess the healing potential of OFM as a novel rotator cuff augmentation graft by thorough in vitro evaluation and assessment in a rat supraspinatus defect model. Clinically, rotator cuff augmentation could lead to reduced re-tear rates, earlier mobilisation of the shoulder and thus reduced joint stiffness and ultimately improved post-operative function.

\section{Methods}

\section{Ethical approval}

The surgical procedure and isolation of primary rat tenocytes were carried out in accordance with the Institutional Animal Ethics Committee. Peripheral blood mononuclear cells were purified from healthy human volunteer blood and collected according to a protocol approved by the Institutional Human Participants Ethics Committee.

\section{Tenocyte cell culture}

Primary rat tenocytes were isolated from tendon fascicles of mature female Wistar rats, as previously described [23]. Briefly, tendon was roughly chopped and digested in dispase and collagenase (both $0.5 \mathrm{mg} / \mathrm{mL}$ from Sigma-Aldrich, USA) in Dulbecco's modified Eagle's medium-F12 (DMEM-F12) with $10 \%$ fetal bovine serum (FBS) at $37{ }^{\circ} \mathrm{C}$ until all the ECM had been digested. The cell suspension was passed through a cell strainer, washed and re-suspended in fresh media. Cells were cultured in $75 \mathrm{~cm}^{2}$ flasks (Corning Inc., USA) with $10 \% \mathrm{FBS} /$ DMEM-F12 and incubated at $37{ }^{\circ} \mathrm{C}$ with $5 \% \mathrm{CO}_{2}$ until confluent.

\section{Scaffold preparation}

OFM was provided by Mesynthes Ltd. (Auckland, New Zealand) as single-ply, lyophilized biomaterial, terminally sterilized with ethylene oxide. Samples were prepackaged as $1.9-\mathrm{cm}$ diameter discs or grafts pre-cut to $5 \times 10 \mathrm{~mm}$.

\section{Qualitative analysis of OFM cytocompatibility}

Primary rat tenocytes, isolated as above, were seeded onto 1.9-cm diameter discs of OFM in 24-well plates (Greiner Bio-One, Germany) for a period of 1,7 and 14 days in DMEM-F12 with $5 \%$ FBS at $2.5 \times 10^{4}$ cells/scaffold. At each time point, scaffolds were stained with calcein AM (Invitrogen) to visualise live cells, as previously described [24]. Scaffolds were washed in phosphate-buffered saline (PBS) and stained for $10 \mathrm{~min}$ at $37^{\circ} \mathrm{C}$ with $2 \mu \mathrm{M}$ calcein 
AM in PBS. Scaffolds were washed in fresh PBS and viewed immediately using fluorescent microscopy; representative images were taken to demonstrate cell density across the scaffold.

\section{Quantitative analysis of OFM cytocompatibility}

Primary rat tenocytes, isolated as above, were seeded as described for the qualitative cytocompatibility assay for a period of 1, 7 and 14 days. At each time point, viability was assessed, as previously described [24]. Briefly, $50 \mu \mathrm{l}$ alamarBlue $^{\odot}(\times 100$, Invitrogen) $(5 \%$ final concentration in well) was added to each well for $4 \mathrm{~h}$ at $37^{\circ} \mathrm{C}$. Following this, $200 \mu \mathrm{l}$ of the alamarBlue ${ }^{\oplus}$ conditioned medium was transferred to a 96-well plate (Greiner Bio-One) and fluorescence read using a Synergy 2 multi-detection microplate reader (BioTek Instruments Inc., USA).

\section{Immunogenicity assay-monocyte-derived dendritic cell activation}

Peripheral blood mononuclear cells were purified from healthy human volunteer blood. Monocytes were isolated from peripheral blood mononuclear cells using the Monocyte Isolation Kit II (Miltenyi Biotec, Germany), according to the manufacturer's protocol. Immature monocytederived dendritic cells were prepared by culturing the monocytes for 6 days in RPMI medium supplemented with $10 \%$ FBS, $100 \mathrm{ng} / \mathrm{mL}$ granulocyte macrophagecolony stimulating factor (GM-CSF) and $50 \mathrm{ng} / \mathrm{mL}$ interleukin-4 (IL-4) (PeproTech, USA).

The immunogenicity of OFM was assessed using immature dendritic cells, as previously described [24]. Cells were plated in 24-well plates containing either $5 \mathrm{ng} / \mathrm{mL}$ lipopolysaccharide (LPS; Sigma-Aldrich) or $1.9-\mathrm{cm}$ diameter OFM in RPMI medium with 10 \% FBS. The expression of cell surface markers associated with dendritic cell maturation was assessed following $40 \mathrm{~h}$ of incubation.

Treated dendritic cells were incubated on ice for $20 \mathrm{~min}$ with mouse anti-human antibodies against PE/Cy7-antiCD80 (clone 2D10), PerCP/Cy5.5 anti-CD83 (clone HB15e) and APC anti-CD86 (clone IT2.2) (all supplied by BioLegend, USA). Excess antibody was removed by washing with PBS containing $1 \%$ FBS. Cell surface markers were analysed by flow cytometry using the FACS ARIA II (BD Biosciences), and data analysis was performed using FloJo (version 7.6) (BD Biosciences).

\section{In vivo assessment}

Male Sprague-Dawley rats $(n=34)$, aged greater than 12 weeks and weighing greater than $350 \mathrm{~g}$, underwent surgery. Rats were checked for general health and randomly distributed into three weight-matched groups: (1) sham surgery (approach to supraspinatus only), $n=10$, (2) unaugmented control (single row supraspinatus repair), $n=12$ and (3) intervention group (single row repair augmented with OFM), $n=12$.

At least $2 \mathrm{~h}$ prior to surgery, rats received a subcutaneous injection of the non-steroidal anti-inflammatory drug (NSAID) Rimadyl (10 $\mu \mathrm{L} / \mathrm{g})$. For anaesthetic induction, rats were placed in a sealed rodent induction box with $5 \%$ isoflurane and $2 \mathrm{~L} \mathrm{O}_{2}$. Anaesthesia was maintained during surgery with $2.5 \%$ isoflurane and $2 \mathrm{~L} \mathrm{O}_{2}$ through a specialised nose cone. A 2.5 -mL subcutaneous injection of normal saline was administered immediately after induction.

The forelimb was prepared by shaving and washing with $2 \%$ chlorhexidine and $70 \%$ ethanol, followed by a sterile drape (Fig. 1a). An approximately 2-cm-longitudinal incision was made, centred over the glenohumeral joint, extending proximally along the belly of supraspinatus and distally along shaft of humerus (Fig. 1b). The middle belly of deltoid was incised proximally, in line with muscle fibres, down to the lateral aspect of the proximal humeral shaft. The acromio-clavicular joint was incised. The supraspinatus was easily identified through this approach (Fig. 1c). A 5-0 prolene stay suture was passed through the distal supraspinatus. The tendon was incised at its insertion onto the greater tuberosity of the humerus (Fig. 1d). The insertion site was debrided of any residual soft tissue (Fig. 1e).

In the unaugmented control group, supraspinatus repair was carried out using the 5-0 prolene stay suture and a modified Mason-Allen suture technique (Fig. 1f). The cut end of the tendon was approximated to the bony insertion, and the suture ends were tied with a surgeon's knot (Fig. 1g). In the intervention group, the OFM scaffolds $(5 \times 10 \mathrm{~mm})$ were rehydrated with sterile saline, then overlaid longitudinally on the superficial aspect of the tendon-bone insertion and incorporated into the suture repair (passes 2-5) (Fig. 1h).

Following repair, the deltoid and coraco-acromial arch were lightly approximated with two interrupted 2-0 vicryl sutures. Skin closure was carried out with a running subcuticular 4-0 monocryl suture with buried knots at either ends. After closure, $0.4 \mathrm{~mL}$ of Marcain $(1.25 \mathrm{mg} / \mathrm{mL}$ solution) local anaesthetic was infiltrated around the operation site.

Rats were closely monitored immediately following surgery. Once sufficiently recovered from the anaesthetic, they were housed singularly and transferred to a warming cabinet for one night. Rimadyl $(10 \mu \mathrm{L} / \mathrm{g})$ and $2-\mathrm{mL}$ normal saline were administered subcutaneously twice daily for $48 \mathrm{~h}$ post-operation. Rats were weighed daily and checked for signs of illness, pain or distress twice daily for the first $48 \mathrm{~h}$ post-operation. Following this, they were weighed and checked once daily until 14 days post-operation. They were then weighed and checked on a weekly basis. 

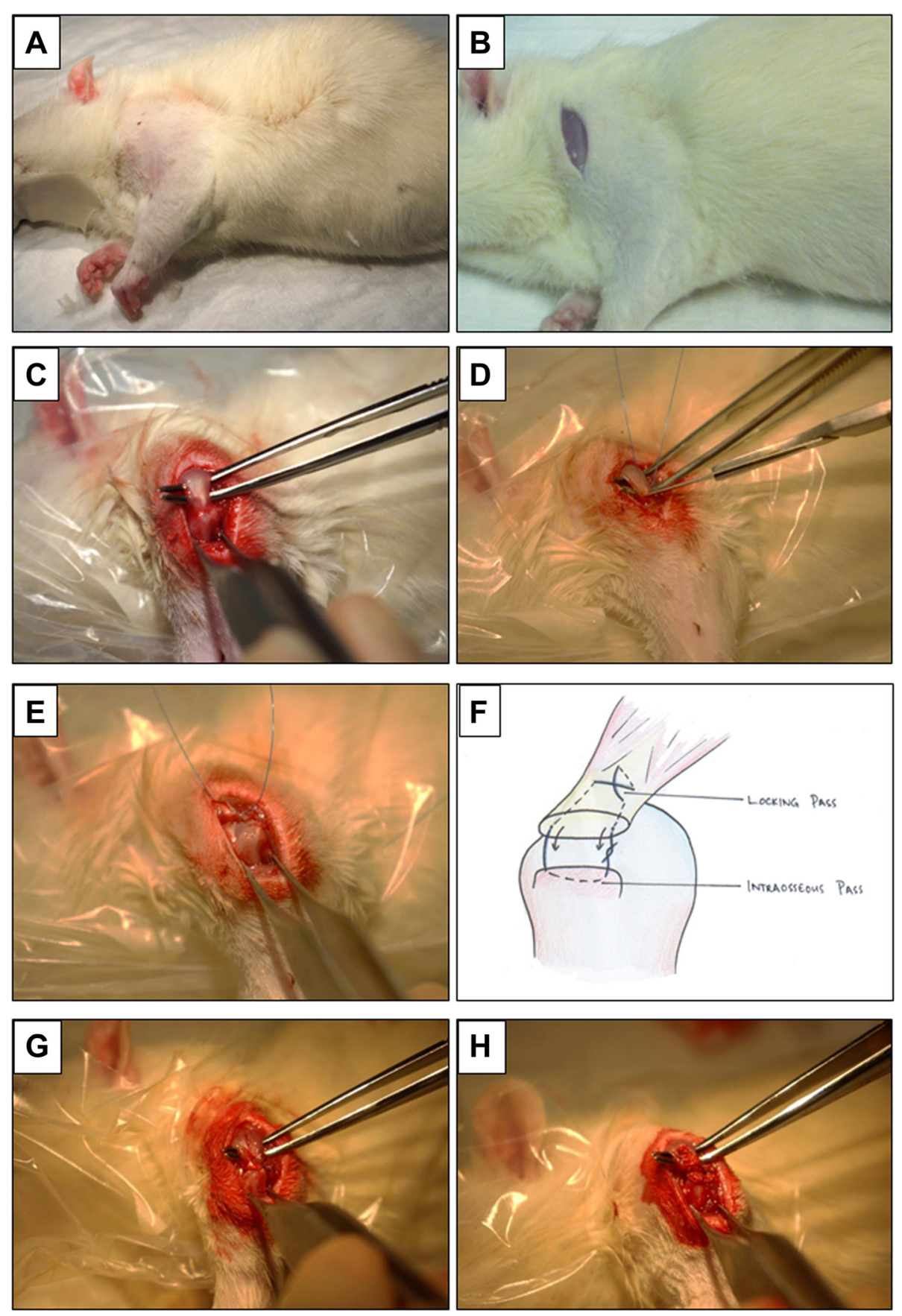

Fig. 1 Intraoperative guide to in vivo surgery. Micrographs representing each stage of the operative procedure including the preparation and positioning of the shoulder (a), creating an incision over the humeral head (b), isolating the exposed supraspinatus (c), transecting the supraspinatus from the humeral head (d), removing residual soft tissue from the humeral head (e), the modified Mason-Allen suture repair (f) and the unaugmented and augmented supraspinatus tendons $(\mathbf{g}, \mathbf{h})$

At either 6 or 12 weeks post-operatively, rats were humanely sacrificed by $\mathrm{CO}_{2}$ inhalation. The left supraspinatus tendon, attached to the whole humerus, was immediately excised and either placed in formalin for histological analysis or wrapped in PBS soaked gauze and stored at $-20^{\circ} \mathrm{C}$ for later biomechanical analysis.

\section{Biomechanical analysis}

Excised shoulders were defrosted in a $37{ }^{\circ} \mathrm{C}$ water bath and kept hydrated with $\mathrm{H}_{2} \mathrm{O}$ spray throughout testing. The muscle fibres of the supraspinatus were removed by gentle scraping, leaving only the distal tendon attached to the humerus. Suture material was 
removed to allow testing of the repaired tendon alone.

Using a specially modified clamp, the humerus and the tendon were positioned in an Instron machine, with the tendon at $45^{\circ}$ relative to the humerus, to apply load in a functional position. Specimens underwent a 10-cycle preconditioning phase $(0.1$ to $0.5 \mathrm{~N}$ at a rate of $1 \% / \mathrm{s})$, followed by a stress relaxation phase $(6 \%$ strain, at a rate of $5 \% / \mathrm{s}(0.575 \mathrm{~mm} / \mathrm{s})$, followed by $10 \mathrm{~min}$ of relaxation, then ramp to failure at a rate of $0.3 \% / \mathrm{s}$. Stiffness, Young's modulus of elasticity and ultimate load to failure were then calculated. For each treatment group, at each time point ( 6 and 12 weeks) $n=4$ samples were tested.

\section{Histological analysis}

Excised shoulders were fixed in $10 \%$ neutral buffered formalin, processed, decalcified in $10 \%$ formic acid $/ 5 \%$ formaldehyde, embedded in paraffin and 5 - $\mu$ m-thick sections taken. Sections were stained with haematoxylin and eosin $(\mathrm{H} \& \mathrm{E})$ and analysed using both transmitted and polarised light microscopy to evaluate healing. A semi-quantitative grading system was used based on collagen fibre density, collagen fibre orientation, quality of healing at bonetendon interface, vascularity and presence of inflammatory cells, as described in Table 1. A total was obtained by combining the scores for these five parameters, with a higher score indicating greater healing. Scoring was carried out by a musculoskeletal histopathologist (MD) and an orthopaedic registrar (MS) who were blinded to treatment details and each other's scores. A minimum of three slides per repair were assessed where the tendon-bone interface could be identified.

\section{Statistical analysis}

Data from both the in vitro and in vivo analyses were analysed using one-way analysis of variance (ANOVA) with post hoc Tukey's test using GraphPad Prism Software (GraphPad Software, San Diego, CA, USA).

\section{Results}

\section{Qualitative and quantitative analysis of OFM}

\section{cytocompatibility}

Cytocompatibility of OFM was confirmed quantitatively by alamarBlue ${ }^{\mathrm{rax}}$ assay of tenocyte viability and qualitatively by calcein AM fluorescent staining of live tenocytes. Quantitative analysis demonstrated that tenocyte cell numbers increase from day 1 to day 7 , after which cell numbers change little up to day 14 . Cell growth was highly variable over the 14-day culture period, as demonstrated by the large error bars. Qualitative analysis demonstrated that primary rat tenocytes successfully adhered to OFM on day 1 and the number of viable cells increased over the 14-day period (Fig. 2).

\section{Immunogenicity assay-monocyte-derived dendritic cell activation}

The immunogenicity of implantable scaffolds has been strongly correlated to the maturation of antigen-presenting dendritic cells, including the upregulation of dendritic cell surface proteins such as CD80, CD83 and CD86.

Following $40 \mathrm{~h}$ of exposure to OFM, dendritic cell expression levels of CD80, CD83 and CD86 were unchanged compared to the low levels expressed by untreated control dendritic cells (Fig. 3). This was in contrast to dendritic cells exposed to LPS, which had elevated levels of all three maturation markers.

\section{In vivo assessment}

All surgeries were uneventful with no anaesthetic or operative complications. Post operatively, rats recovered rapidly, returning to full use of the operated limb within 48-72 h. All 34 animals survived until the experimental endpoints, and no hypersensitivity reactions were observed. Furthermore, macroscopic inspection of the collected specimens did not reveal any gross infectious or inflammatory changes.

\section{Biomechanical analysis}

An important outcome required when augmenting a rotator cuff repair is for the biomechanical performance of the tendon to replicate its pre-injury state allowing appropriate forces across the repair to aid healing by improving collagen orientation. In order to assess this, the repaired rotator cuffs were biomechanically tested along a functional axis, with stiffness, elasticity and total load to failure measured. Biomechanical analysis revealed no significant differences in stiffness, elasticity or load to failure at either time points.

At 6 weeks post-repair, the stiffness and elasticity of the augmented rotator cuffs were higher than the unaugmented controls, although not significant (Fig. 4). Stiffness was measured at $11.8+/-2.2 \mathrm{~N} / \mathrm{mm}$ in the augmented group,

Table 1 Histological grading system used to determine the rotator cuff healing outcomes

\begin{tabular}{llllll}
\hline & Collagen fibre density & Collagen fibre orientation & Bone-tendon interface & Vascularity & Inflammation \\
\hline 0 & None & None & $0-24 \%$ interdigitation & Abundant vascular network & Abundant inflammatory cells \\
1 & Low & Disorganised fibres & $25-49 \%$ interdigitation & Moderate vascular network & Moderate inflammatory cells \\
2 & Medium & Moderate alignment & $50-75 \%$ interdigitation & Minimal vascular network & Minimal inflammatory cells \\
3 & High & Highly aligned & $>75 \%$ interdigitation & No vascular network & No inflammatory cells \\
\hline
\end{tabular}



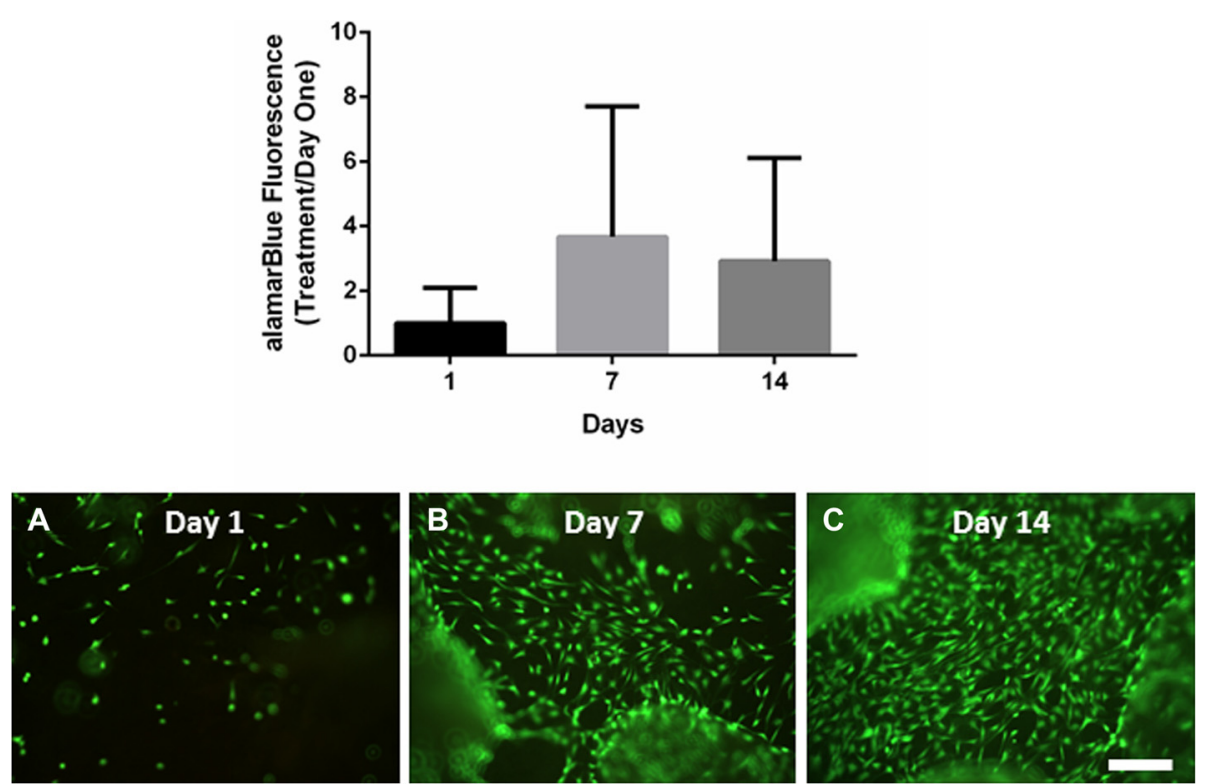

Fig. 2 OFM supports primary rat tenocyte growth. Cytocompatibility analysis of primary rat tenocytes cultured on OFM for 1,7 and 14 days using alamarBlue ${ }^{\mathrm{TM}}$ assay and calcein AM fluorescent staining (a day 1, b day 7, $\mathbf{c}$ day 14). Scale bar $=200 \mu \mathrm{m}$. Observations were performed three times. Data presented are representative of these biological repeats. $N=3$

compared to $9.2+/-2.9 \mathrm{~N} / \mathrm{mm}$ in the unaugmented group, and Young's modulus of elasticity measured at 18.8 $+/-5.8 \mathrm{MPa}$ for the augmented group and 8.2+/-2.3 $\mathrm{MPa}$ for the unaugmented group. Similarly, there was no significant difference between the unaugmented group and the sham group, which had a mean stiffness of $10.4+/-4.8 \mathrm{~N} /$ $\mathrm{mm}$ and a mean elasticity of $10.5+/-2.9 \mathrm{MPa}$.

At 12 weeks, there were similarly no differences in the stiffness and elasticity of all groups. Stiffness was measured at $11.9+/-1.6 \mathrm{~N} / \mathrm{mm}$ for the augmented group, $9.1+/-2.2 \mathrm{~N} / \mathrm{mm}$ for the unaugmented group and $13.9 \mathrm{~N} /$ $\mathrm{mm}$ for the sham-operated group. The Young's modulus of elasticity measured was measured at $12.7+/-0.82 \mathrm{MPa}$ for the augmented group, $14.9+/-3.4 \mathrm{MPa}$ for the unaugmented group and 11.3 MPa for the sham-operated group.

There were no statistically significant differences in the load to failure of the repaired rotator cuffs. The mean load to failure was $20+/-2.8 \mathrm{~N}$ in the augmented group and $19+/-3.8 \mathrm{~N}$ in the unaugmented group at 6 weeks postrepair, and $23+/-2.4 \mathrm{~N}$ and $15+/-2.9 \mathrm{~N}$ at 12 weeks postrepair, respectively. The load to failure of the sham group was $17+/-3.8 \mathrm{~N}$ at 6 weeks and $25+/-6.6 \mathrm{~N}$ at 12 weeks.

\section{Histological analysis}

At 6 weeks post-op, histological scoring of the repaired rotator cuffs showed no difference between the unaugmented group and the OFM augmented group, with both scoring 5.25 out of 15 (Table 2). The sham-operated group scored significantly higher at 11 out of 15 (Table 2).
Histological analysis at 12 weeks post-op showed the sham-operated group to have a well-defined tendonbone interface, with the collagen fibres of the tendon appearing dense and well organised (Fig. 5a, b); this was represented by a histological score of 10.75 (out of 15).

In the unaugmented group, while there was evidence of the initial stages of an intact tendon-bone interface, the tendon fibres appeared frayed and disorganised in nature (Fig. $5 \mathrm{c}, \mathrm{d}$ ), and they therefore scored significantly lower than the sham group in the histological scoring (4.25 out of 15$)$.

The rotator cuffs that had been augmented with OFM had a well-formed tendon-bone interface and a dense collagen fibre network at the tendon; however, the fibres were largely disorganised and the tenocyte cells present had a rounded, non-tenocyte-like morphology (Fig. 5e, f). There was no significant difference in the histological score of the OFM group (7 out of 15) compared to either the sham group or the unaugmented group.

\section{Discussion}

Here, we have shown that the novel ovine forestomach ECM scaffold material is cytocompatible to tenocyte cell growth, non-immunogenic and improves the histological appearance of the rotator cuff when used as an overlay augment in a pre-clinical model of rotator cuff repair.

Tears of the rotator cuff are a common, often painful and a debilitating problem [1]. Current rotator cuff repairs aim to re-attach the tendon to the humeral head and debride a partial tear or suture a full thickness tear 


\section{OFM}
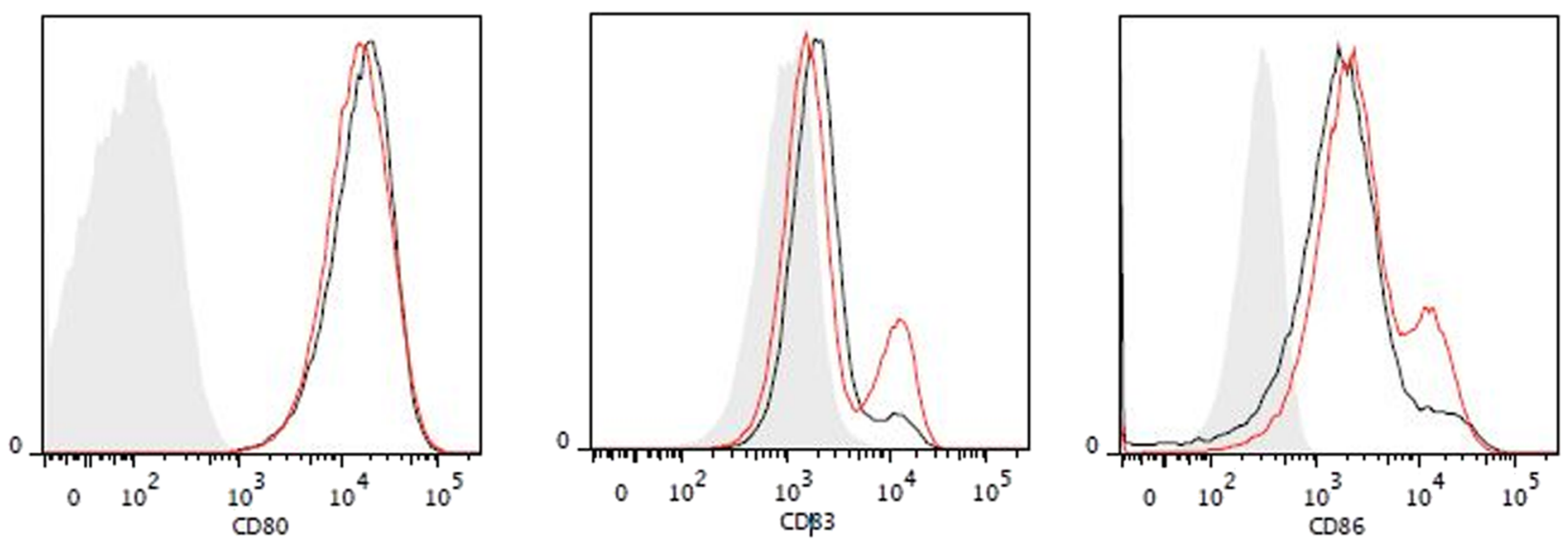

\section{LPS}
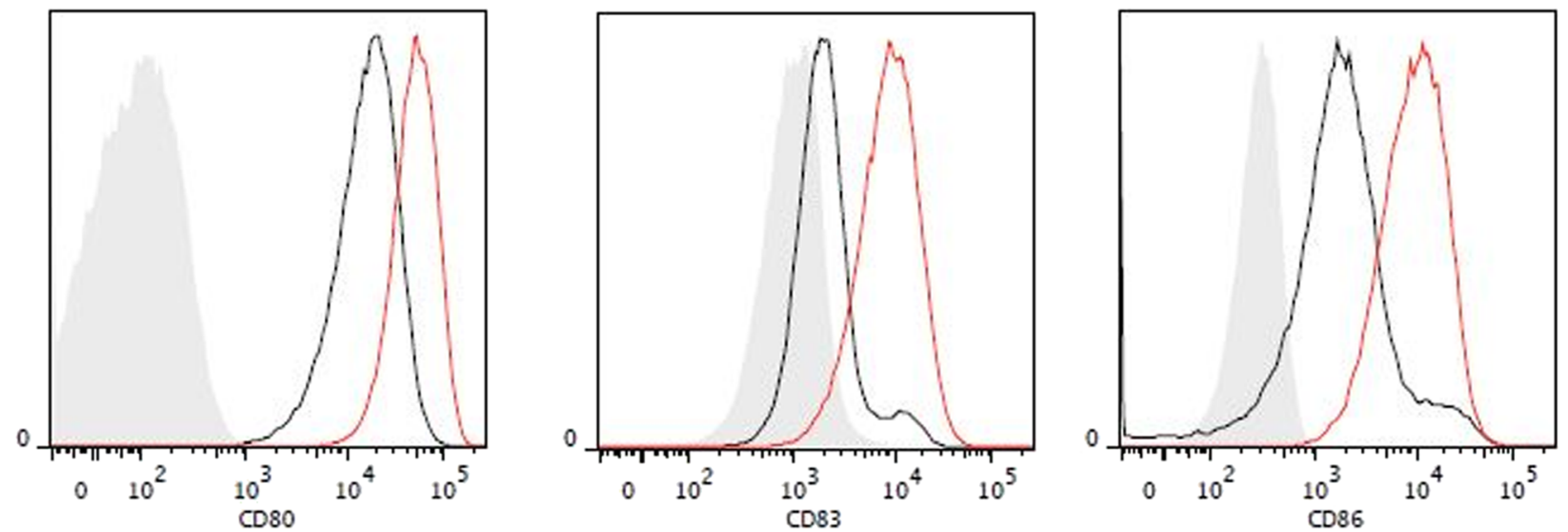

Fig. 3 OFM does not promote dendritic cell maturation. Representative FACS plots of CD80, CD86 and CD83 expression, as markers of dendritic cell maturation, following $40 \mathrm{~h}$ of culture with OFM and LPS. The shaded area represents the unstained control, the black line is the untreated control and the red line represents the treatment group as indicated. Three different patient samples were analysed, each experiment run in duplicates. Data presented are from one patient as representative for the three patients. $N=3$

using open or arthroscopic surgeries. While these techniques can be successful, disorganised or incomplete healing is common [7-9].

Previously, autografts from the biceps tendon or fascia lata have been trialled as interpositional grafts for improving rotator cuff repair surgical outcomes $[25,26]$ as have allograft rotator cuffs [27]. However, these methods have had varying results and have not been adopted as common practice. Biomaterial scaffold augmentation has therefore been suggested as a means to enhance the healing outcomes for small to large rotator cuff tears (grade 3 or 4) or when repairing a degenerative tendon [10].

According to a recent study, there were 13 FDA-approved biomaterial scaffolds available for use in augmenting rotator cuff repair. Of these, nine were ECM-based materials, three were synthetic and one was an ECM-woven polymer hybrid [10]. There are limited clinical studies on these materials, some suggesting that these scaffolds improve clinical outcomes; however, the results are generally inconclusive due to a lack of appropriate controls [28-30]. This leads to the consensus that currently available scaffolds, while promising, fail to meet clinical needs [10].

ECM-based scaffolds have been produced from many sources including dermis, small intestinal submucosa, pericardium, Achilles tendon and fascia lata of human, bovine, porcine or equine origin [11,31]. OFM is an ECM scaffold produced from ovine forestomach. Cellular debris is removed using a novel decellularisation technique that maintains more of the natural structure of the ECM and thus improves the hosts healing response [17]. Previous studies have shown that OFM is non-toxic, supports mammalian cell growth and differentiation in vitro and 
6 weeks
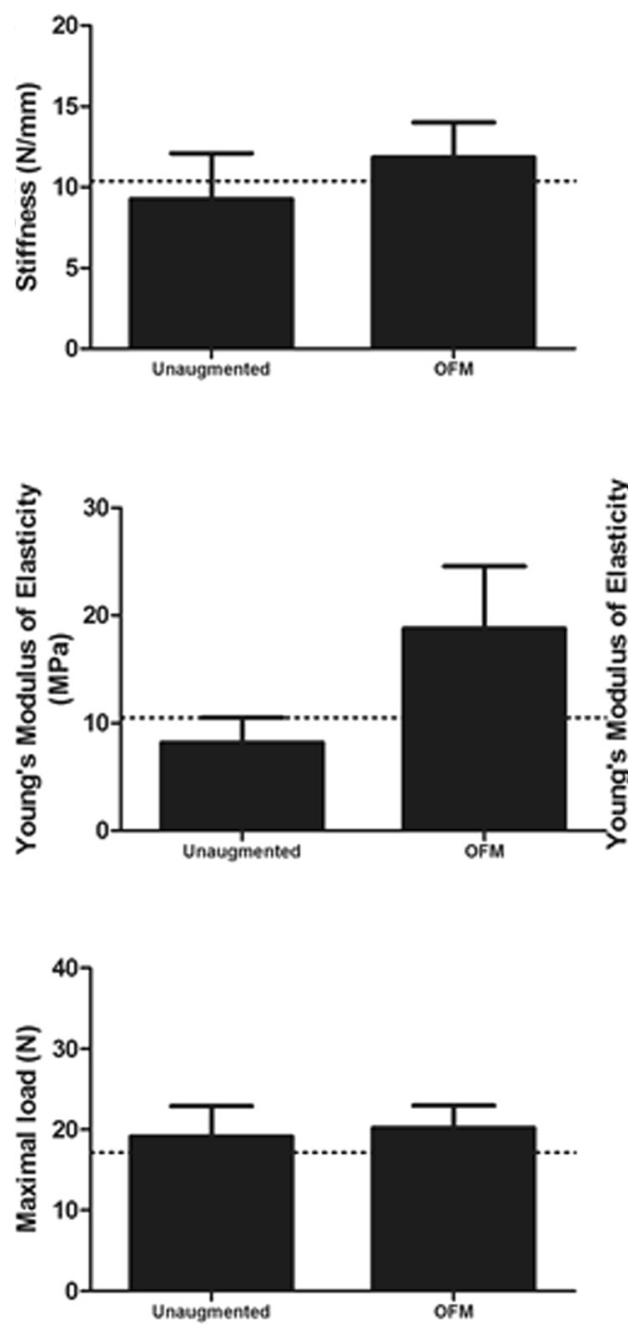

12 weeks
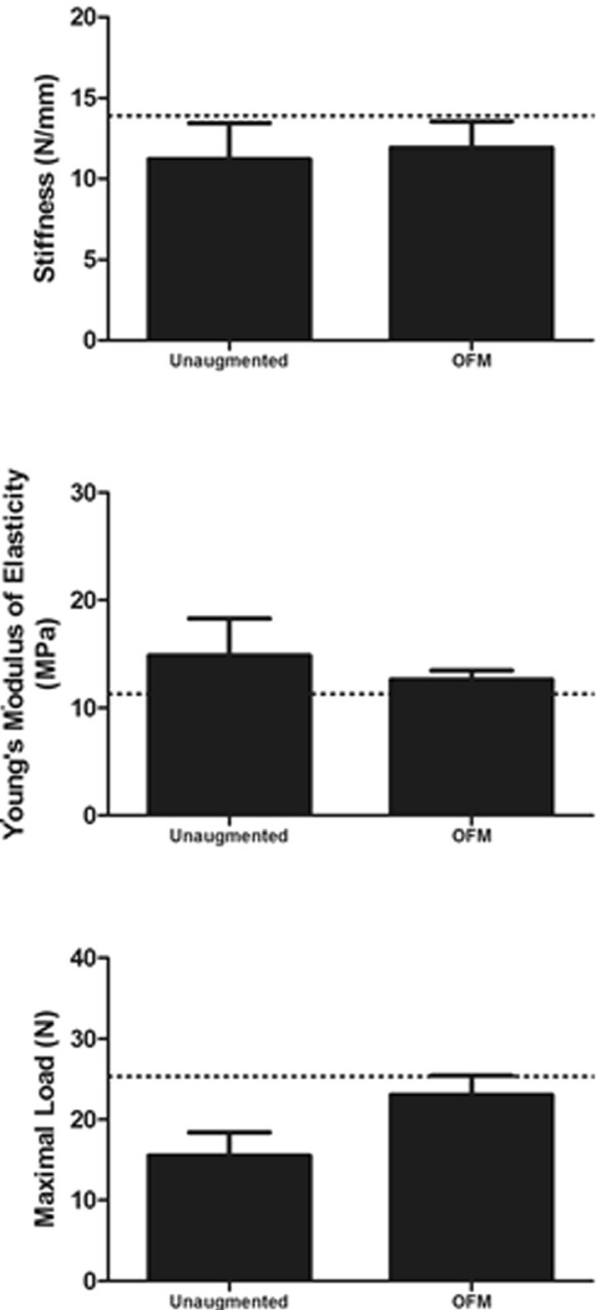

Fig. 4 The biomechanical profiles of repaired rotator cuffs do not differ post-operatively. Mean stiffness, elasticity and maximal load to failure of unaugmented and OFM augmented rat shoulders at 6 and 12 weeks post-surgery. Dotted line indicates the mean measurements of sham-operated shoulders as a reference. $N=4$

Table 2 Histological grading of healed rotator cuffs, as scored by a specialist musculoskeletal pathologist and an orthopaedic registrar based on the criteria set out in Table 1

\begin{tabular}{lllllll}
\hline Group & Collagen fibre density & Collagen fibre orientation & Bone-tendon interface & Vascularity & Inflammation & Total \\
\hline 6 weeks & & & & & & \\
$\quad$ Sham & $2.5( \pm 0.29)$ & $2.5( \pm 0.29)$ & $2.5( \pm 0.29)$ & $2.5( \pm 0.29)$ & $2.5( \pm 0.29)$ & $11( \pm 0.91)$ \\
Unaugmented & $1.5( \pm 0.5)$ & $0.75^{*}( \pm 0.48)$ & $1( \pm 0.71)$ & $11^{*}( \pm 0.41)$ & $1^{*}( \pm 0)$ & $5.25^{*}( \pm 1.60)$ \\
OFM augmentation & $1.25( \pm 0.25)$ & $1.25( \pm 0.25)$ & $1( \pm 0.65)$ & $0.25^{*}( \pm 0.25)$ & $1^{*}( \pm 0)$ & $5.25^{*}( \pm 0.85)$ \\
12 weeks & & & $2( \pm 0.71)$ & $1.25( \pm 0.48)$ & $2( \pm 0)$ & $10.75( \pm 1.44)$ \\
Sham & $2.75( \pm 0.25)$ & $2.75( \pm 0.25)$ & $0.5( \pm 0.5)$ & $0.5( \pm 0.29)$ & $1.25( \pm 0.25)$ & $4.25^{*}( \pm 1.60)$ \\
Unaugmented & $1.5( \pm 0.5)$ & $0.5^{*}( \pm 0.29)$ & $2( \pm 0.41)$ & $0.25( \pm 0.25)$ & $1.5( \pm 0.29)$ & $7( \pm 1)$ \\
OFM augmentation & $2( \pm 0)$ & $1.25^{*}( \pm 0.25)$ & & &
\end{tabular}

Data presented are the mean of both scorers \pm SEM. One-way ANOVA with post hoc Tukey's test. $N=2$ ${ }^{*} p<0.05$ compared to sham 

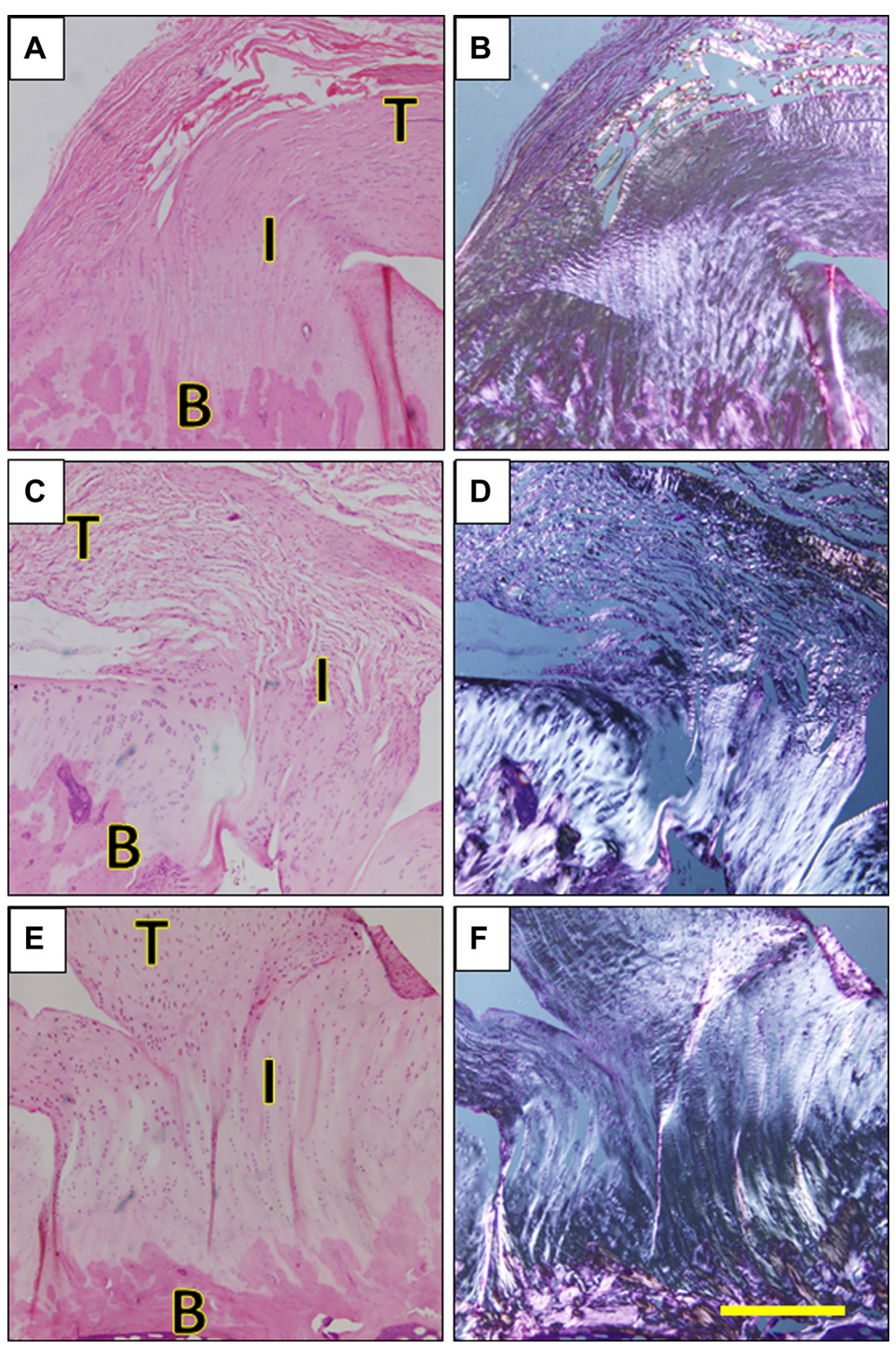

Fig. 5 OFM improves healing of the tendon-bone interface. Representative micrographs of healing at the tendon-bone interface at 12 weeks post-operatively stained with haematoxylin and eosin and viewed under transmitted light (a, c, e) (left) and polarised light (b, d, f) (right). a, b Sham-operated shoulders. c, d Unaugmented control shoulders. e, f OFM augmented shoulders. Bone (B), tendon (T), tendon-bone interface (I). Scale bar represents $400 \mathrm{~mm} . \mathrm{N}=2$

enhances angiogenesis ex vivo and in vivo [17, 32]. Here, we have similarly shown that OFM supports the adherence and growth of primary tenocyte cells in vitro, although there did appear to be some variance between repeat experiments.
OFM has previously been used successfully in clinical and in vivo studies of wound healing [18-20, 22]; however, given the history of ECM-based materials in producing hypersensitivity reactions in patients undergoing rotator cuff repair [14-16], we feel that prior to pre- 
clinical animal models, it is important to assess the immunogenicity of novel biomaterials. Previous studies have demonstrated that maturation of monocyte-derived dendritic cells in response to biomaterials is indicative of in vivo inflammatory responses [33-35]. In this study, we demonstrated that OFM does not enhance the maturation of primary human dendritic cells in vitro and is therefore unlikely to illicit an adverse response when implanted in vivo.

In order to provide translational evidence that a novel material is a suitable scaffold, capable of improving the surgical outcomes of rotator cuff repair, pre-clinical assessment is required in a well-defined animal model of rotator cuff repair. Many animal models have been used to investigate rotator cuff pathology and repair [36]. Unlike the rabbit or sheep, the rat has a supraspinatus tendon enclosed under a bony arch, with a wide range of motion similar to that of humans, making the rat the recommended model for such procedures [36]. Both extrinsic and intrinsic insults to the supraspinatus tendon cause histological and biomechanical deterioration [4, 36], allowing the model to be used to mimic a wide range of rotator cuff pathologies and to investigate novel treatments for these pathologies. Here, we found the rat model to be relatively simple and reproducible, as well as practically and financially viable.

Augmentation with a biomaterial scaffold aims to both provide mechanical support to the repair and improve the quality of tendon healing [11]. In this study, there were no differences in the biomechanical properties (stiffness, elasticity and load to failure) between the unaugmented or OFM augmented rotator cuffs. The single-ply lyophilized OFM, used in this study, has relatively low mechanical strength compared to the native tendon (load to failure of 15.07 $\mathrm{N}$ and suture pull-out of $5.91 \mathrm{~N}$ ). However, OFM biomaterial has been fabricated into multi-ply presentations, with load to failures of over $65 \mathrm{~N}$ [37], allowing scope for a more mechanically robust augment through tailoring of the fabrication procedure. However, these were not practical to use in this study due to the relative size of the rat supraspinatus.

Using overlay augmentation with the OFM scaffold, we showed improved histological outcomes compared to animals treated with standard surgical repairs and no significant difference in histological scoring between the OFM augmented tendons and the sham controls. Augmented tendons had improved collagen fibre density and orientation, and improved quality of healing at the tendon-bone interface. Importantly, at neither time points was there evidence of a hyper-inflammatory response to OFM, as shown by low vascularity and a low presence of inflammatory cells.

Previous studies have demonstrated that ECM-based materials induce healing responses by directing macrophage response down an $\mathrm{M} 2$, pro-remodelling phenotype, rather than the pro-inflammatory M1 phenotype [38]. Given the improvements in collagen fibre organisation and the lack of inflammatory responses seen in the histological sections, it would be of interest to determine what effect OFM has on macrophage response in tendon repair, although this was out of the scope of the present study. Similarly, determining how human tenocytes respond to OFM would be an important step in future translation from bench to bedside. Here, rat tenocytes were used as these are directly relevant to the pre-clinical model being employed.

\section{Conclusion}

In conclusion, there is a clear clinical need for improving surgical outcomes of rotator cuff repair; however, the ideal biomaterial augment has not been identified or clinically implemented as yet. Here, we have shown that OFM as an overlay augment improves the histological appearance of the rotator cuff post-surgical healing but does not provide any mechanical benefit in its current iteration.

Overall, ECM scaffolds offer a promising solution to a difficult clinical problem. Identifying materials that offset the poorer mechanical properties of the rotator cuff post-injury/repair and enhance organised tendon healing still remain paramount to incorporating augmentation into current rotator cuff surgical procedures.

\section{Competing interests}

The authors declare that they have no competing interests.

\section{Authors' contributions}

All authors have made contributions to the current work. The manuscript was written by MS and DSM and critically revised by all authors. The design of the study was done by DSM, BC, JC and MS. The in vitro cytocompatibility assessment was carried out by DSM and the immunogenicity assay by JDM and $\mathrm{KB}$ under the supervision of PRD. The surgical procedure and postoperative care was carried out by MS, DT and KEC. Mechanical testing was carried out by AT and MS. Histology was carried out by SA and DSM and analysed by MS and MD. The final data was interpreted by all authors. All authors read and approved the final manuscript.

\section{Acknowledgements}

This study was funded by an Auckland Medical Research Foundation Postdoctoral Fellowship for D.S. Musson (1 311 002), the Health Research Council of New Zealand (12/1110), supported by the Medical Technologies Centre of Research Excellence (MedTech CoRE), funded by the Tertiary Education Commission of New Zealand and by the European Union Seventh Framework Programme skelGEN (FP7/2007-2013) under grant agreement number [318553]. The funders took no part in the analyses or in the writing of this manuscript

\section{Author details}

'Department of Medicine, The University of Auckland, Private Bag 92019, Auckland 1142, New Zealand. 'Faculty of Engineering, University of Auckland, Auckland 1142, New Zealand. ${ }^{3}$ Waikato District Health Board, Waikato Hospital, Hamilton 3204, New Zealand. ${ }^{4}$ Department of Anatomy with Radiology, The University of Auckland, Auckland 1142, New Zealand. ${ }^{5}$ School of Biological Sciences, The University of Auckland, Auckland 1142, New Zealand. ${ }^{6}$ Maurice Wilkins Centre, University of Auckland, Private Bag 92014, Auckland, New Zealand. ${ }^{7}$ Department of Orthopaedics, Middlemore Hospital, Private Bag 93311, Auckland, New Zealand. 
Received: 6 August 2015 Accepted: 11 October 2015

Published online: 20 October 2015

\section{References}

1. Chakravarty K, Webley M. Shoulder joint movement and its relationship to disability in the elderly. J Rheumatol. 1993;20(8):1359-61.

2. Yamamoto A, Takagishi K, Osawa T, Yanagawa T, Nakajima D, Shitara H, et al. Prevalence and risk factors of a rotator cuff tear in the general population. J Shoulder Elbow Surg. 2010;19(1):116-20. doi:10.1016/ j.jse.2009.04.006.

3. Ensor KL, Kwon YW, Dibeneditto MR, Zuckerman JD, Rokito AS. The rising incidence of rotator cuff repairs. J Shoulder Elbow Surg. 2013;22(12):1628-32. doi:10.1016/j.jse.2013.01.006

4. Carpenter JE, Thomopoulos S, Flanagan CL, DeBano CM, Soslowsky LJ. Rotator cuff defect healing: a biomechanical and histologic analysis in an animal model. J Shoulder Elbow Surg. 1998;7(6):599-605.

5. Kartus J, Kartus C, Rostgard-Christensen L, Sernert N, Read J, Perko M. Longterm clinical and ultrasound evaluation after arthroscopic acromioplasty in patients with partial rotator cuff tears. Arthroscopy. 2006;22(1):44-9. doi:10.1016/j.arthro.2005.07.027.

6. Massoud SN, Levy O, Copeland SA. Subacromial decompression. Treatment for small- and medium-sized tears of the rotator cuff. J Bone Joint Surg. 2002;84(7):955-60.

7. Boileau P, Brassart N, Watkinson DJ, Carles M, Hatzidakis AM, Krishnan SG. Arthroscopic repair of full-thickness tears of the supraspinatus: does the tendon really heal? J Bone Joint Surg Am. 2005;87(6):1229-40. doi:10.2106/ JBJS.D.02035.

8. DeHaan AM, Axelrad TW, Kaye E, Silvestri L, Puskas B, Foster TE. Does double-row rotator cuff repair improve functional outcome of patients compared with single-row technique? A systematic review. Am J Sport Med. 2012;40(5):1176-85

9. Duquin TR, Buyea C, Bisson L. Which method of rotator cuff repair leads to the highest rate of structural healing? A systematic review. Am J Sports Med. 2010;38(4):835-41. doi:10.1177/0363546509359679.

10. Derwin KA, Badylak SF, Steinmann SP, lannotti JP. Extracellular matrix scaffold devices for rotator cuff repair. J Shoulder Elbow Surg. 2010;19(3):467-76. doi:10.1016/j.jse.2009.10.020.

11. Ricchetti ET, Aurora A, lannotti JP, Derwin KA. Scaffold devices for rotator cuff repair. J Shoulder Elbow Surg. 2012;21(2):251-65. doi:10.1016/ j.jse.2011.10.003.

12. Badylak S, Obermiller J, Geddes L, Matheny R. Extracellular matrix for myocardial repair. Heart Surg Forum. 2003;6(2):E20-6.

13. van der Rest M, Garrone R. Collagen family of proteins. FASEB J. 1991;5(13):2814-23.

14. lannotti JP, Codsi MJ, Kwon YW, Derwin K, Ciccone J, Brems JJ. Porcine small intestine submucosa augmentation of surgical repair of chronic two-tendon rotator cuff tears. A randomized, controlled trial. J Bone Joint Surg Am. 2006;88(6):1238-44. doi:10.2106/JBJS.E.00524.

15. Walton JR, Bowman NK, Khatib Y, Linklater J, Murrell GAC. Restore orthobiologic implant: not recommended for augmentation of rotator cuff repairs. J Bone Joint Surg-Am Vol. 2007;89A(4):786-91.

16. Zheng MH, Chen J, Kirilak Y, Willers C, Xu J, Wood D. Porcine small intestine submucosa (SIS) is not an acellular collagenous matrix and contains porcine DNA: possible implications in human implantation. J Biomed Mater Res B Appl Biomater. 2005;73(1):61-7. doi:10.1002/jbm.b.30170.

17. Lun S, Irvine SM, Johnson KD, Fisher NJ, Floden EW, Negron L, et al. A functional extracellular matrix biomaterial derived from ovine forestomach Biomaterials. 2010;31(16):4517-29. doi:10.1016/j.biomaterials.2010.02.025.

18. Bohn GA, Gass K. Leg ulcer treatment outcomes with new ovine collagen extracellular matrix dressing: a retrospective case series. Adv Skin Wound Care. 2014;27(10):448-54. doi:10.1097/01.ASW.0000453728.12032.6f.

19. Liden BA, May BC. Clinical outcomes following the use of ovine forestomach matrix (endoform dermal template) to treat chronic wounds. Adv Skin Wound Care. 2013;26(4):164-7. doi:10.1097/ 01.ASW.0000428862.34294.d4.

20. Simcock JW, Than M, Ward BR, May BC. Treatment of ulcerated necrobiosis lipoidica with ovine forestomach matrix. J Wound Care. 2013;22(7):383-4. doi:10.12968/jowc.2013.22.7.383.

21. Merrilees MJ, Falk BA, Zuo N, Dickinson ME, May BC, Wight TN. Use of versican variant $\mathrm{V} 3$ and versican antisense expression to engineer cultured human skin containing increased content of insoluble elastin. J Tissue Eng Regen Med. 2014. doi:10.1002/term.1913.

22. Simcock J, May BC. Ovine forestomach matrix as a substrate for single-stage split-thickness graft reconstruction. Eplasty. 2013;13:e58.

23. Chhana A, Callon KE, Dray M, Pool B, Naot D, Gamble GD, et al. Interactions between tenocytes and monosodium urate monohydrate crystals: implications for tendon involvement in gout. Ann Rheum Dis. 2014;73(9):1737-41. doi:10.1136/annrheumdis-2013-204657.

24. Musson DS, Naot D, Chhana A, Matthews BG, McIntosh JD, Lin ST, et al. In vitro evaluation of a novel non-mulberry silk scaffold for use in tendon regeneration. Tissue Eng Part A. 2015;21(9-10):1539-51. doi:10.1089/ten.TEA.2014.0128.

25. Ito J, Morioka T. Surgical treatment for large and massive tears of the rotator cuff. Int Orthop. 2003;27(4):228-31. doi:10.1007/s00264-003-0459-4.

26. Neviaser JS. Ruptures of the rotator cuff of the shoulder. New concepts in the diagnosis and operative treatment of chronic ruptures. Arch Surg. 1971;102(5):483-5.

27. Moore DR, Cain EL, Schwartz ML, Clancy Jr WG. Allograft reconstruction for massive, irreparable rotator cuff tears. Am J Sports Med. 2006;34(3):392-6. doi:10.1177/0363546505281237.

28. Barber FA, Burns JP, Deutsch A, Labbe MR, Litchfield RB. A prospective, randomized evaluation of acellular human dermal matrix augmentation for arthroscopic rotator cuff repair. Arthroscopy. 2012;28(1):8-15. doi:10.1016/ j.arthro.2011.06.038.

29. Burkhead WZ, Schiffern SC, Krishnan SG. Use of graft jacket as an augmentation for massive rotator cuff tears. Semin Arthroplasty. 2007;18(1):11-8. doi:10.1053/j.sart.2006.11.017.

30. Wong I, Burns J, Snyder S. Arthroscopic graft jacket repair of rotator cuff tears. J Shoulder Elbow Surg. 2010;19(2 Suppl):104-9. doi:10.1016/j.jse.2009.12.017.

31. Badylak SF. The extracellular matrix as a biologic scaffold material. Biomaterials. 2007;28(25):3587-93. doi:10.1016/j.biomaterials.2007.04.043.

32. Irvine SM, Cayzer J, Todd EM, Lun S, Floden EW, Negron L, et al. Quantification of in vitro and in vivo angiogenesis stimulated by ovine forestomach matrix biomaterial. Biomaterials. 2011;32(27):6351-61. doi:10.1016/j.biomaterials.2011.05.040.

33. Foti M, Granucci F, Pelizzola M, Beretta O, Ricciardi-Castagnoli P. Dendritic cells in pathogen recognition and induction of immune responses: a functional genomics approach. J Leukoc Biol. 2006;79(5):913-6. doi:10.1189/Jlb.1005547.

34. Norton LW, Park J, Babensee JE. Biomaterial adjuvant effect is attenuated by anti-inflammatory drug delivery or material selection. J Control Release. 2010;146(3):341-8. doi:10.1016/j.jconrel.2010.05.032.

35. Kou PM, Pallassana N, Bowden R, Cunningham B, Joy A, Kohn J, et al. Predicting biomaterial property-dendritic cell phenotype relationships from the multivariate analysis of responses to polymethacrylates. Biomaterials. 2012;33(6):1699-713. doi:10.1016/j.biomaterials.2011.10.066.

36. Soslowsky L, Carpenter JE, DeBano CM, Banerji I, Moalli MR. Development and use of an animal model for investigations on rotator cuff disease. J Shoulder Elbow Surg. 1996;5(5):383-92.

37. Floden EW, Malak SF, Basil-Jones MM, Negron L, Fisher JN, Lun S, et al. Biophysical characterization of ovine forestomach extracellular matrix biomaterials. J Biomed Mater Res B Appl Biomater. 2011;96(1):67-75. doi:10.1002/jbm.b.31740.

38. Badylak SF, Valentin JE, Ravindra AK, McCabe GP, Stewart-Akers AM. Macrophage phenotype as a determinant of biologic scaffold remodeling. Tissue Eng Part A. 2008;14(11):1835-42. doi:10.1089/ten.tea.2007.0264.

\section{Submit your next manuscript to BioMed Central and take full advantage of:}

- Convenient online submission

- Thorough peer review

- No space constraints or color figure charges

- Immediate publication on acceptance

- Inclusion in PubMed, CAS, Scopus and Google Scholar

- Research which is freely available for redistribution 